\title{
MATERIALS SCIENCE APPLICATIONS OF A MONOCHROMATED TEM
}

\author{
G.A. Botton*, S. Lazar, M.-Y.Wu, F.D. Tichelaar, H.W. Zandbergen
}

National Centre for HREM, Department of Technical Sciences, Delft University of Technology, Delft, The Netherlands, *Permanent Address: Brockhouse Institute of Materials Research, McMaster University, Hamilton, Ont. L8S 4M1, Canada

With the realization of the first field emission transmission electron microscope equipped with a high resolution EELS monochromator ${ }^{[1]}$ it becomes possible to explore potential applications of the system in materials science. The improved energy resolution on a TEM opens new possibilities for studying detailed electronic structure and bonding effects evaluated from near-edge structure features and dielectric function measurements via the low-loss part of the spectrum. For the latter, these improvements are particularly significant for the direct measurement of band gaps as these can be carried out locally on features of interest in nanoscale structures.

For the EELS experiments presented here a FEI Tecnai-200FEG monochromator was used. This system is equipped with a pre-specimen monochromator and a high-resolution Gatan Imaging Filter (GIF) for EELS ${ }^{[1,2]}$. With this setup it is possible to obtain a $100 \mathrm{meV}$ energy resolution using a sub $2 \mathrm{~nm}$ probe or $500 \mathrm{meV}$ with a $0.20 \mathrm{~nm}$ probe. It is therefore possible to use the scanning module of the microscope to obtain high-resolution line profiles. The first report with extensive materials science application of the system ${ }^{[3]}$ showed the improvements of the spectral resolution even for $\mathrm{K}$ edges of light elements and the study of $\mathrm{GaN}$ defects with high-spatial resolution. The application of this system to the study of interfacial layers in high-dielectric constant materials has also been demonstrated recently ${ }^{[4]}$.

Other applications of the system for core-loss near edge structures at the $\mathrm{Ti}_{23}$ edges in $\mathrm{BaTiO}_{3}$ and $\mathrm{SrTiO}_{3}$ are shown in Fig. 1. It is possible to clearly visualize the subtle changes in the spectrum due to the differences in the local Ti coordination imposed by the change from a tetragonal structure in $\mathrm{BaTiO}_{3}$ to the cubic structure in $\mathrm{SrTiO}_{3}$. Spectra obtained with XAS show the comparable and well-resolved $t_{2 g}$ and $e_{g}$ splitting for the $\mathrm{SrTiO}_{3} \mathrm{Ti} \mathrm{L}_{23}$ edge. The triplet states at the edge threshold are also clearly visible (Figure 1b).

In the low-loss regime of the EELS spectrum, the applications of a TEM with monochromator are potentially more significant as the tails of the zero-loss peak are dramatically reduced. An example of the low-loss spectrum for BaTiO3 is shown in Figure 2. The intensity onset is extremely well resolved from the tails of the zero-loss peak. In addition, the energy of the onset at about $3.6 \mathrm{eV}$ is in good agreement with the optical value. It is possible to obtain accurate measurement of the bandgap using quantitative fitting of the low-loss data and also to follow the dispersion of the interband transitions ${ }^{[3]}$ by tuning the scattering vector $\mathbf{q}$ in the experiments (Figure 3$)^{[5]}$.

[1] P.C. Tiemeijer, J.H.A. van Lin, A.F. de Jong, Microscopy and Microanalysis 7, suppl 2 (2001), $1130-1131$.

[2] H.A. Brink, M. Barfels, B. Edwards, and P. Burgner, Microscopy and Microanalysis 7, Suppl. 2 (2001) 908-909.

[3] S. Lazar, G.A. Botton, M.-Y.Wu, F.D. Tichelaar, H.W. Zandbergen, In Press, Ultramicroscopy, SALSA special issue, R.D. Leapman Editor.

[4] G.A. Botton et al., In press, Electrochemical Society Proceedings, Paris 2003

[5] This work is supported by NSERC (Canada) and by the Government of The Netherlands. 

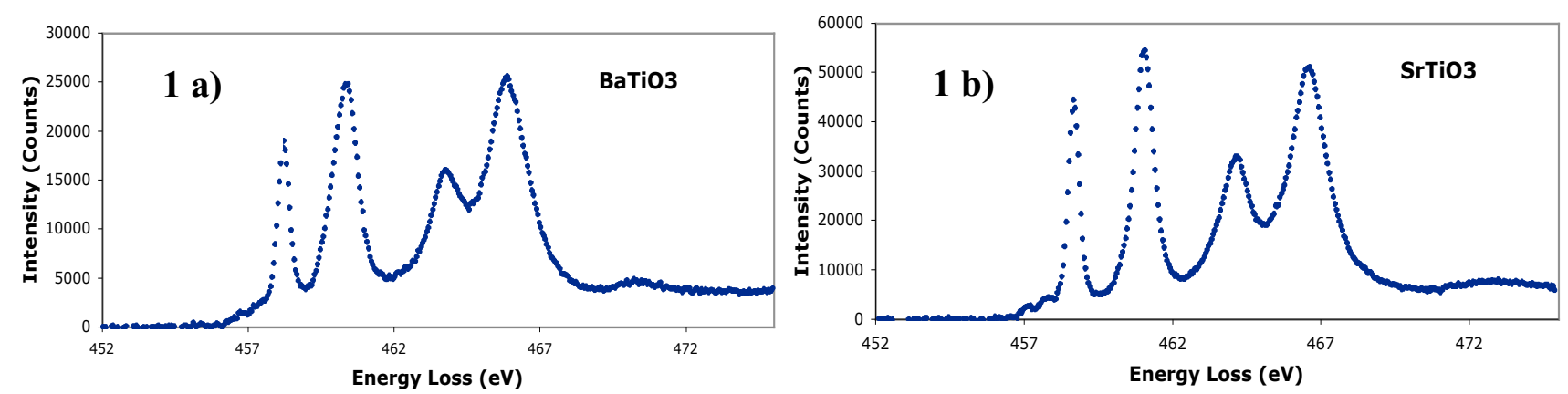

Figure 1. Ti $\mathrm{L}_{23}$ edges in $\mathrm{BaTiO}_{3}(\mathrm{a})$ and $\mathrm{SrTiO}_{3}$ (b). Differences due to distortions of the local Ti environment are clearly resolved. The full width half maximum of the $\mathrm{L}_{3} \mathrm{t}_{2 \mathrm{~g}}$ peak in $\mathrm{SrTiO}_{3}$ is $0.4 \mathrm{eV}$.

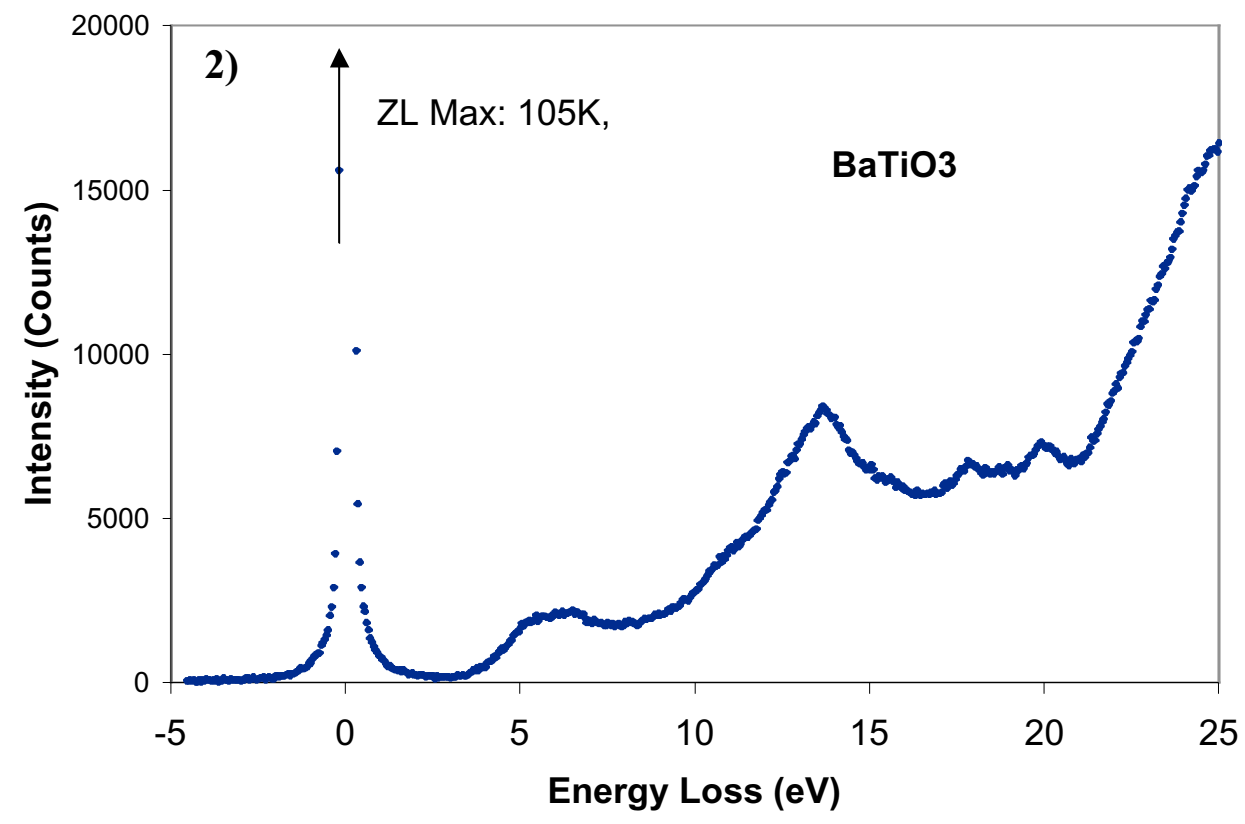

Figure 2. Low -loss spectrum of $\mathrm{BaTiO}_{3}$. The intensity onset is well resolved from the tails of the zeroloss peak. The maximum intensity of the zero-loss peak is $105 \mathrm{~K}$ counts.

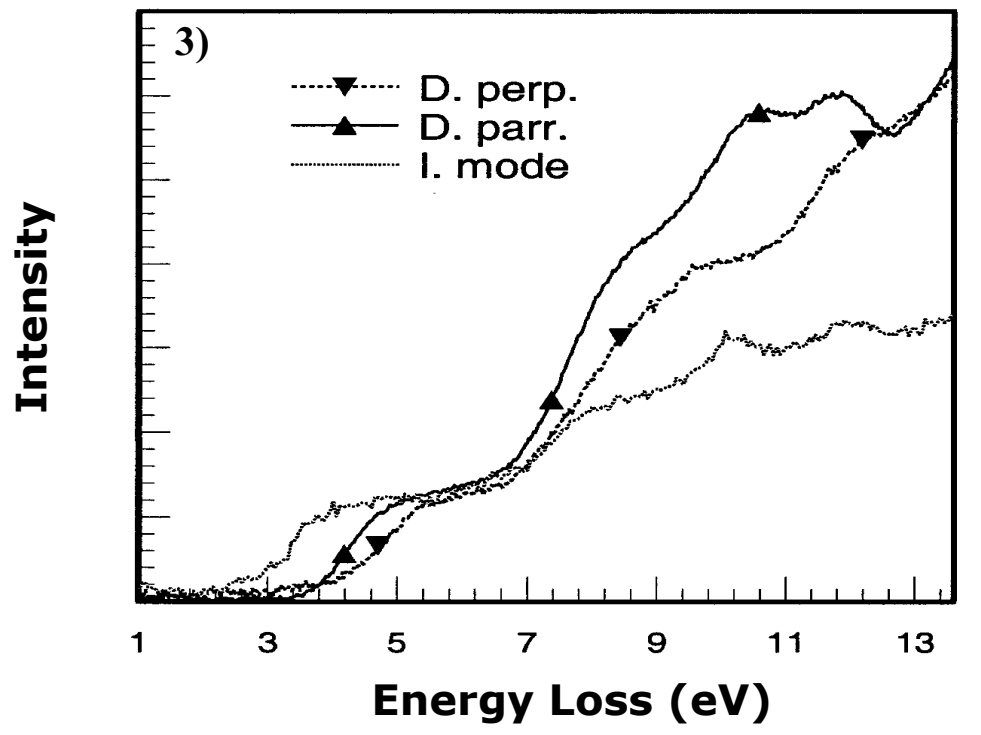

Figure 3. Low-loss spectrum of GaN obtained in image mode (I.mode) and in diffraction mode with momentum transfer vector $\mathbf{q}$ parallel to the $\mathrm{C}$ axis of the $\mathrm{GaN}$ crystal (D. par.) and with $\mathbf{q}$ perpendicular to the $\mathrm{C}$ axis (D. perp). The differences in the bandgap values (over $1 \mathrm{eV}$ ) induced by forcing non-vertical transitions are clearly visible. Differences in the interband transitions at about $10 \mathrm{eV}$ are enhanced when forcing non-vertical transitions. 